Article

\title{
Green Synthesis and Characterization of Gold Nanoparticles Using Lignin Nanoparticles
}

\author{
Baobin Wang ${ }^{1}$, Guihua Yang ${ }^{1, * \mathbb{C}}$, Jiachuan Chen ${ }^{1}$ and Guigan Fang ${ }^{2, *}$ \\ 1 State Key Laboratory of Biobased Material and Green Papermaking, Qilu University of \\ Technology (Shandong Academy of Sciences), Jinan 250353, China; wangbaobin0408@163.com (B.W.); \\ chenjc@qlu.edu.cn (J.C.) \\ 2 Institute of Chemical Industry of Forest Products, Chinese Academy of Forestry, Nanjing 210042, China \\ * Correspondence: ygh2626@126.com (G.Y.); fangguigan@icifp.cn (G.F.)
}

Received: 11 August 2020; Accepted: 16 September 2020; Published: 18 September 2020

\begin{abstract}
With The development of nanotechnology, gold nanoparticles (Au NPs) have attracted enormous attention due to their special properties. The green synthesis of Au NPs from lignin would inspire The utilization of lignin and its related functional materials. In this study, a rapid preparation process of Au NPs was investigated by utilizing lignin nanoparticles (LNPs) under room temperature without chemical addition. The LNPs acted as a reducing agent, stabilizing agent, and template for The preparation of LNPs@AuNPs. The obtained LNPs@AuNPs were characterized by UV-Vis spectrum, Transmission Electron Microscope (TEM), and X-ray photoelectron spectroscopy (XPS). The possible mechanism was illustrated by Fourier Transform Infrared Spectroscopy (FT-IR), ${ }^{31} \mathrm{P}$, XPS, and UV analyses. The abundant hydroxyl groups $(24.96 \mathrm{mmol} / \mathrm{g})$ favored The preparation of $\mathrm{Au}$ NPs. Au NPs diameters of 10-30 nm were well dispersed in The LNPs. The optimal reaction conditions were a ratio of $10 \mathrm{mg}$ of LNPs to $0.05 \mathrm{mmol} \mathrm{HAuCl}_{4}$, room temperature, and a reaction time of $30 \mathrm{~min}$. The LNPs@AuNPs exhibited excellent stability in The suspension for more than seven days. The reduction process could be related to The disruption of side chains of lignin, hydroxyl group oxidation, and hydroquinones and quinones from The comproportionation reaction. The LNPs@AuNPs would open a door for The design of Au NP/lignin-derived novel functional materials.
\end{abstract}

Keywords: lignin nanoparticles (LNPs); gold nanoparticles (Au NPs); green synthesis; nanotechnology; functional material

\section{Introduction}

With The development of nanotechnology and nanoscience, metal nanoparticles have attracted enormous attention because of their novel optical/electrical properties [1,2]. Metal nanoparticles, especially gold nanoparticles (Au NPs), are used as multifunctional gold nanoparticle-derived materials for various applications, including photothermal conversion [3,4], simultaneous detection and imaging [4,5], and targeted chemo-photothermal treatments [6], etc. Due to The versatility of The Au NPs, several strategies have been applied for The synthesis of Au NPs, such as irradiation reduction [7], thermal decomposition [8], and chemical reduction [9]. The most common method for The preparation of Au nanoparticles is chemical reduction. However, The chemical reduction process usually requires additional chemical addition (reducing agents, stabilizing agents, and surfactants) and harsh conditions which violate The basic principles of green chemistry [10]. Thus, a more environmentally friendly procedure is needed to prepare The Au NPs.

Lignin, The most abundant aromatic, is a biopolymer derived from trees and crops [11]. It is a three-dimensional amorphous polyphenol which consists of methoxylated phenylpropane 
substructures. Nowadays, millions of tons of industrial lignin are obtained from pulp mills; however, The lignin is normally used as fuel during The pulping process which is a rather inefficient way to utilize The lignin [11]. Extensive studies are devoted to exploit value-added applications, such as cosmetics, water treatment, adhesive, soil treatment, food, and medicine, etc. [12,13]. In The past decade, researchers found that The 3D structure and abundant surface groups (hydroxyl, carbonyl, and aldehyde groups) could act as reducing and stabilizing agents for The green synthesis of metal nanoparticles. Various approaches were applied in The green synthesis of metal nanoparticles including microwave irradiation, mechanical grinding method, and heat reduction. Han et al. reported $\mathrm{Au}$ NP-loaded liquid marble using lignin as a reducing agent under microwave radiation at $80^{\circ} \mathrm{C}$ for $60 \mathrm{~min}$ [14]. The liquid marble exhibited excellent properties for The detection of $\mathrm{Hg}^{2+}$ and photothermal conversion. Lin et al. reported The green synthesis of Au NPs with The addition of hemicellulose/lignin at $100{ }^{\circ} \mathrm{C}$ [15]. With The development of material science, The regular structure of nanomaterials is essential to The design of functional materials for advanced applications. Moreover, The recent progress in The preparation of lignin nanoparticles (LNPs) inspired The design of lignin-based functional materials [16-18]. Thus, The green synthesis of well-dispersed Au NPs with LNPs is important for The preparation of composite lignin-derived materials.

Herein, LNPs were utilized as reducing and stabilizing agents for The green synthesis of well-dispersed LNPs@AuNPs under room temperature. The Au NPs were well dispersed in The LNPs due to The stabilizing effect of The lignin. Au NPs were confined in The LNP structure which would facilitate The design of The lignin-based materials. The total green synthesis of Au NPs was conducted under room temperature and without chemical addition which is consistent with The principles of green chemistry. Moreover, The reducing mechanism and reaction behavior were analyzed by ${ }^{31}$ P-NMR, FTIR, XPS, UV-Vis spectrum, and TEM. The possible mechanism was also proposed.

\section{Materials and Methods}

\subsection{Materials}

$\mathrm{HAuCl}_{4} \cdot 3 \mathrm{H}_{2} \mathrm{O}$ was purchased from Sigma-Aldrich Co, Ltd. Ethanol (analytical grade) was purchased from Fisher, Co, Ltd. Lignin from prehydrolysis liquor was obtained through acid precipitation. The $\mathrm{pH}$ value of The prehydrolysis liquor was buffered to 2 . Then, $10 \mathrm{~g}$ of prehydrolysis lignin (PL) was mixed with ethanol $(300 \mathrm{~mL})$ and stirred for $12 \mathrm{~h}$. The solution was filtered, and The purified lignin was recovered by vacuum evaporation. All other reagents were used without further purification.

\subsection{Preparation of $L N P$}

The LNPs were prepared following The anti-solvent method [19]. Specifically, $0.1 \mathrm{~g}$ lignin was added to $150 \mathrm{~mL}$ ethanol under magnetic stirring at $300 \mathrm{rpm}$ for $2 \mathrm{~h}$. Then, $70 \mathrm{~mL}$ deionized water was poured into The lignin/ethanol mixture, and The mixed solution was stirred for $4 \mathrm{~h}$ until The solution became a uniform solution. The solution was dialyzed in a dialysis bag (molecular weight: 12,000-14,000, Sigma-Aldrich Co, Ltd.) for seven days. The water was changed several times. Finally, The solution was freeze-dried for further characterization.

\subsection{Preparation of $A u N P s @ L N P s$}

LNPs $(10 \mathrm{mg})$ were added to $10 \mathrm{~mL}$ of different concentrations of $\mathrm{HAuCl}_{4}(0.5 \mathrm{mmol} / \mathrm{L}, 2 \mathrm{mmol} / \mathrm{L})$, and The mixture was sonicated in iced water for $10 \mathrm{~min}$ with magnetic stirring at $300 \mathrm{rpm}$. The obtained suspension was transferred to $20 \mathrm{~mL}$ glass vials under room temperature for $30 \mathrm{~min}$. The samples were named LNPs@AuNPs 1 and LNPs@AuNPs 2, respectively. LNPs (10 mg) were added to $10 \mathrm{~mL} 0.5 \mathrm{mmol} / \mathrm{L} \mathrm{HAuCl}_{4}$, and The mixture was sonicated in iced water for $10 \mathrm{~min}$ with magnetic stirring at $300 \mathrm{rpm}$. The obtained suspension was heated at $80^{\circ} \mathrm{C}$ for $30 \mathrm{~min}$ in $20 \mathrm{~mL}$ glass vials. The sample was named LNPs@AuNPs 3. After The reaction completed, The suspension was 
centrifuged at 10,000 rpm for $10 \mathrm{~min}$. The precipitation was washed with deionized water three times, and The obtained LNPs@AuNPs were used for further characterization. All The experiments were performed in duplicate.

\subsection{Characterization}

NMR analysis was processed with a Bruker AVIII $400 \mathrm{MHz}$ spectrometer (Bruker, Karlsruhe, Germany) at room temperature. Phosphorous nuclear magnetic resonance ( ${ }^{31} \mathrm{P}$ NMR) was used to quantitatively determine The functional groups of The lignin, and The detection of The subunits in lignin was performed through two-dimensional heteronuclear single quantum coherence (2D HSQC) NMR spectra. FTIR spectroscopy (NICOLET iS5, Thermo Fisher Scientific, Waltham, MA, USA) was used to characterize The chemical structure of lignin and The composites. The spectra were recorded in The range of $4000-500 \mathrm{~cm}^{-1}$ with a resolution of $4 \mathrm{~cm}^{-1}$ and 32 scans. The $\mathrm{pH}$ values were measured by a pH meter (ALTON pH510 Series). The morphology of LNPs and The composites was observed by a transmission electron microscope (TEM, JEOL 2011, Japan) with a $200 \mathrm{kV}$ acceleration voltage. The samples were individually dispersed in water with sonication before being transferred to a carbon-coated copper grid and left to air-dry at room temperature overnight. Atomic force microscopy (AFM) was used to observe The morphology of LNPs. X-ray diffraction (XRD) patterns were recorded using a Bruker D8 Advance diffractometer with $\mathrm{Cu}$ Ka radiation (40 kV, $30 \mathrm{~mA})$ at a scanning speed of $6^{\circ} / \mathrm{min}$ in The $2 \theta$ range of $10-85^{\circ}$. The element composition of LNPs and The composites was performed using X-ray photoelectron spectroscopy (XPS) (ESCALa-b220i-XL Thermo Fisher Scientific K-Alpha, Thermo Fisher Scientific, Waltham, MA, USA). For The analysis of The XPS peaks, The C 1s and $\mathrm{Au} 4 \mathrm{f}$ position were set at $284.6 \mathrm{eV}$ and $87.6 \mathrm{eV}$, respectively. Inductively coupled plasma atomic emission spectrometry (ICP-AES, Thermo Scientific ICAP 6500, Thermo Fisher Scientific, Waltham, MA, USA) was used to measure The gold content of composites after digestion with hydrochloric and nitric acid $(3: 1, v / v)$ [20]. The reduction process of Au NPs was recorded at room temperature using UV-Vis spectroscopy (Evolution 201, Thermo Fisher Scientific, Waltham, MA, USA). The characteristic peak of Au NPs is at $540 \mathrm{~nm}$.

\section{Results}

\subsection{Schematic of Preparing The LNPs@AuNPs}

The LNPs@AuNPs were prepared through in situ reduction of gold nanoparticles onto LNPs under room temperature (Figure 1I). The PL-based LNPs were prepared through The anti-solvent method. The PL was dissolved in ethanol first, and then deionized water was added as an anti-solvent to obtain LNPs. The LNPs were formed due to intramolecular hydrogen bonding and $\pi-\pi$ stacking of The lignin structure [21]. The green synthesis of Au NPs with LNPs was achieved by directly mixing $\mathrm{HAuCl}_{4}$ solution with LNPs. The Au NPs were reduced at room temperature with The reducing groups (hydroxyl and aldehyde groups) on The lignin structure (Figure 1II). With The increased concentration of $\mathrm{HAuCl}_{4}$ addition, The color of The LNPs@AuNP suspension turned red which is due to The SPR effect of The Au NPs. Moreover, LNPs acted as a stabilizer during The reduction process, and Au NPs were well dispersed in The LNPs. The Au NP suspension was stable even after seven days, which is due to The electrostatic repulsion of The negatively charged surface of the LNPs. 


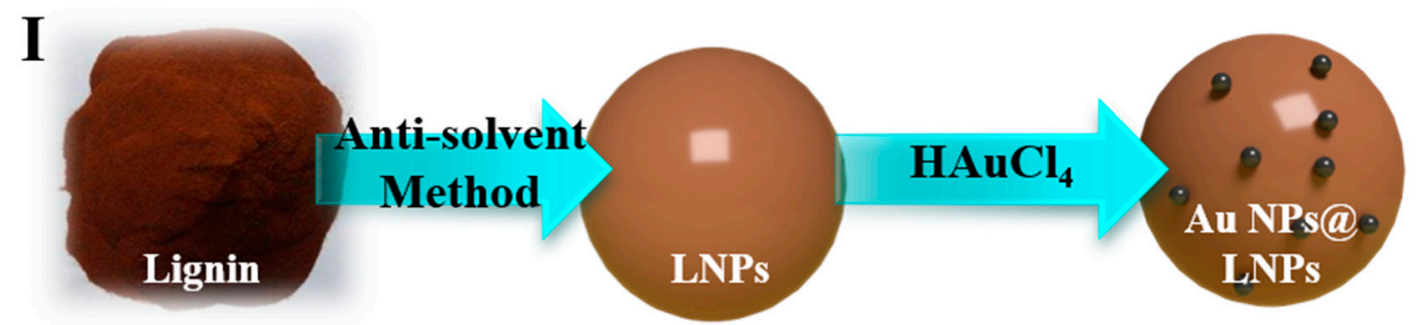

II

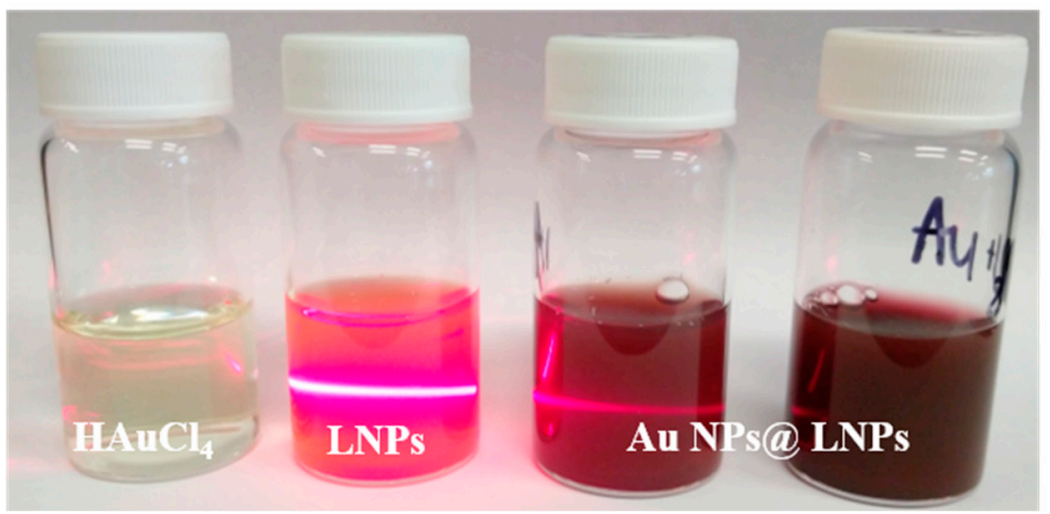

Figure 1. Schematics of The preparation of LNPs@AuNPs. (I). Preparation process of The LNPs@AuNPs, (II). Digital photo of The $\mathrm{HAuCl}_{4}, \mathrm{LNPs}, \mathrm{LNPs} @ A u N P s$.

\subsection{Characterization of Lignin}

The green synthesis of Au NPs was related to The surface functional groups of The lignin; thus, it is essential to analyze The chemical structure of The lignin. The chemical structure of The lignin was characterized with FTIR and ${ }^{31} \mathrm{P}$ spectra. Figure 2 shows The ${ }^{31} \mathrm{P}$ spectrum of The PL. The main $\mathrm{OH}$ groups (syringyl and guaiacyl $-\mathrm{OH}$ ) were observed in The PL which is The typical structure of lignin. The total $-\mathrm{OH}$, noncondensed phenolic $-\mathrm{OH}$ and carboxylic acid contained 24.96, 14.47 and $2.34 \mathrm{mmol} \mathrm{g}^{-1}$ lignin, respectively (Table 1). After The reduction process, The content of The aliphatic and phenolic hydroxyl groups decreased, and The content of The carboxylic groups increased. The results indicated The reduction process may be related to The oxidation of hydroxyl groups. The abundant noncondensed phenolic hydroxyl groups favored The preparation of Au NPs.

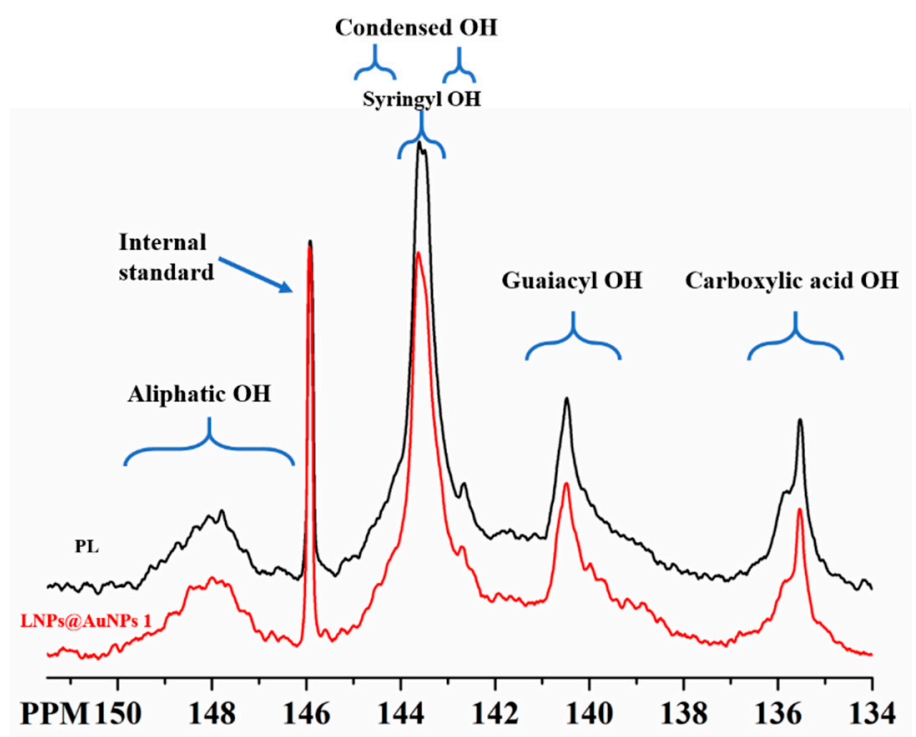

Figure 2. ${ }^{31} \mathrm{P}$ of The PL and LNPs@AuNPs 1. 
Table 1. Quantitative analysis of ${ }^{31} \mathrm{P}$ of PL.

\begin{tabular}{cccccc}
\hline Samples & Aliphatic $\mathbf{O H}$ & Condensed $\mathbf{O H}$ & Syringyl $\mathbf{O H}$ & Guaiacyl $\mathbf{O H}$ & Carboxylic $\mathbf{O H}$ \\
\hline PL & 2.54 & 5.78 & 8.66 & 5.81 & 2.17 \\
LNPs@AuNPs & 2.36 & 4.97 & 6.37 & 4.10 & 2.34 \\
\hline
\end{tabular}

FTIR was used to further characterize The chemical structure of The LNPs and LNPs@AuNPs (Figure 3). The characteristic peak at $3300 \mathrm{~cm}^{-1}$ can be assigned to The phenolic hydroxyl and aliphatic hydroxyl groups of The lignin structure. The peak at $3000-2800 \mathrm{~cm}^{-1}$ is related to The methyl and methylene groups [14]. The peak around 1600-1500 $\mathrm{cm}^{-1}$ can be assigned to The vibration of benzene rings. The peak in The range of $1300-1200 \mathrm{~cm}^{-1}$ is related to $\mathrm{C}-\mathrm{O} / \mathrm{C}=\mathrm{O}$ groups. Also, The peak at $1660 \mathrm{~cm}^{-1}$ is related to The conjugated carbonyl groups. The peak intensity of hydroxyl groups $\left(3300 \mathrm{~cm}^{-1}, 1212 \mathrm{~cm}^{-1}\right)$ decreased after The reduction process which indicated The important role of hydroxyl groups in The preparation of Au NPs. These results demonstrated that LNPs possessed abundant hydroxyl and aldehyde groups which guaranteed The successful preparation of Au NPs [22]. The minor shift of hydroxyl groups that occurred with The LNPs@AuNPs showed The interactions between LNPs and Au NPs.

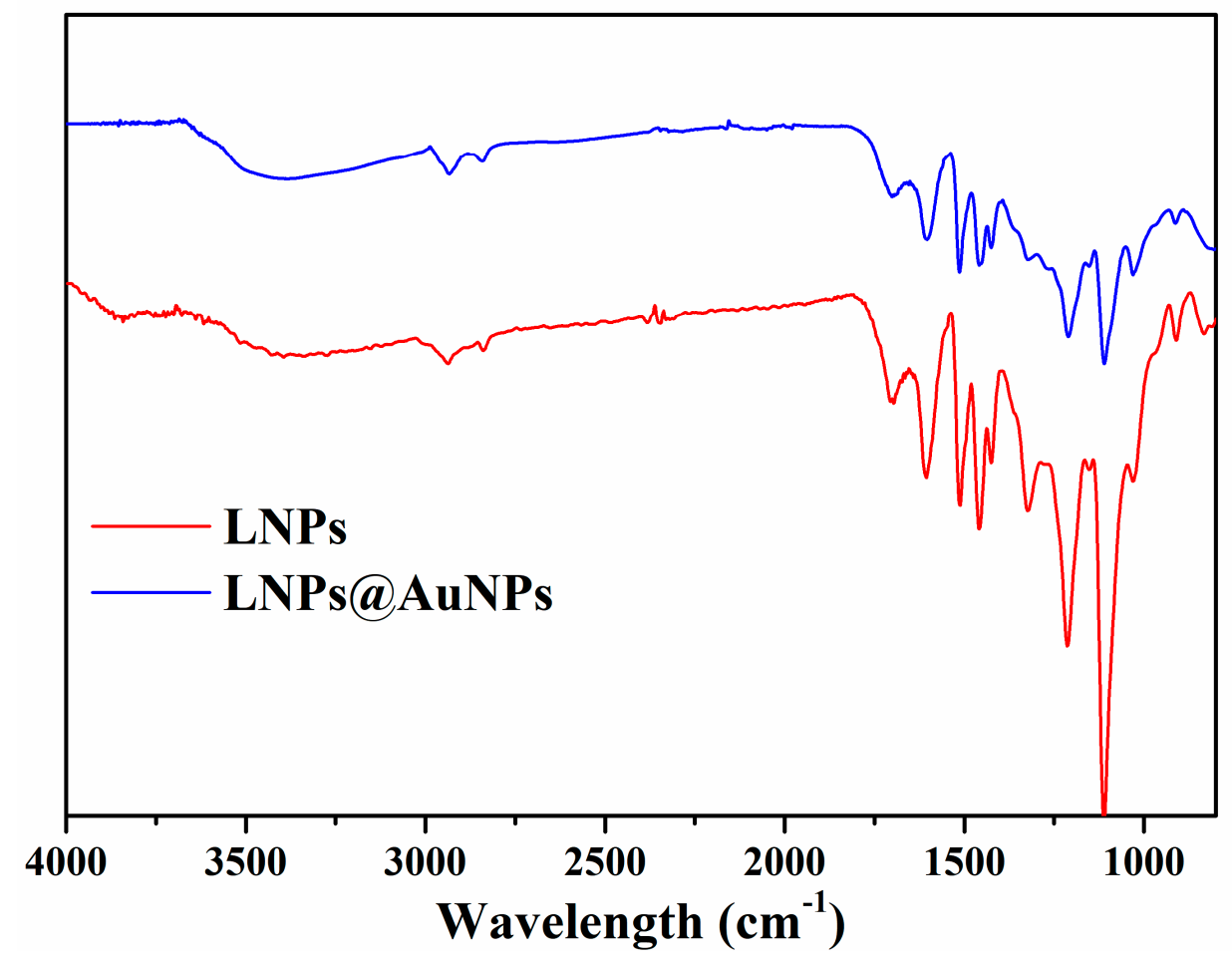

Figure 3. FTIR spectra of The LNPs and LNPs@AuNPs.

Figure 4 shows The hydrodynamic size and zeta potential of The LNPs with different $\mathrm{pH}$ values. DLS results demonstrated that LNPs were stable with a large $\mathrm{pH}$ range, and The hydrodynamic size of The LNPs was around $70 \mathrm{~nm}$. The zeta potential of The negatively charged LNPs increased with The increase in $\mathrm{pH}$ which is due to The deprotonation process. Figure 5 shows The stability of The LNPs during long-term storage. The LNPs were stable over 30 days. The composites aggregated gradually after 10 days due to The decreased zeta potential which might be related to The association between The negatively charged LNPs and ionic species in The aqueous media. 


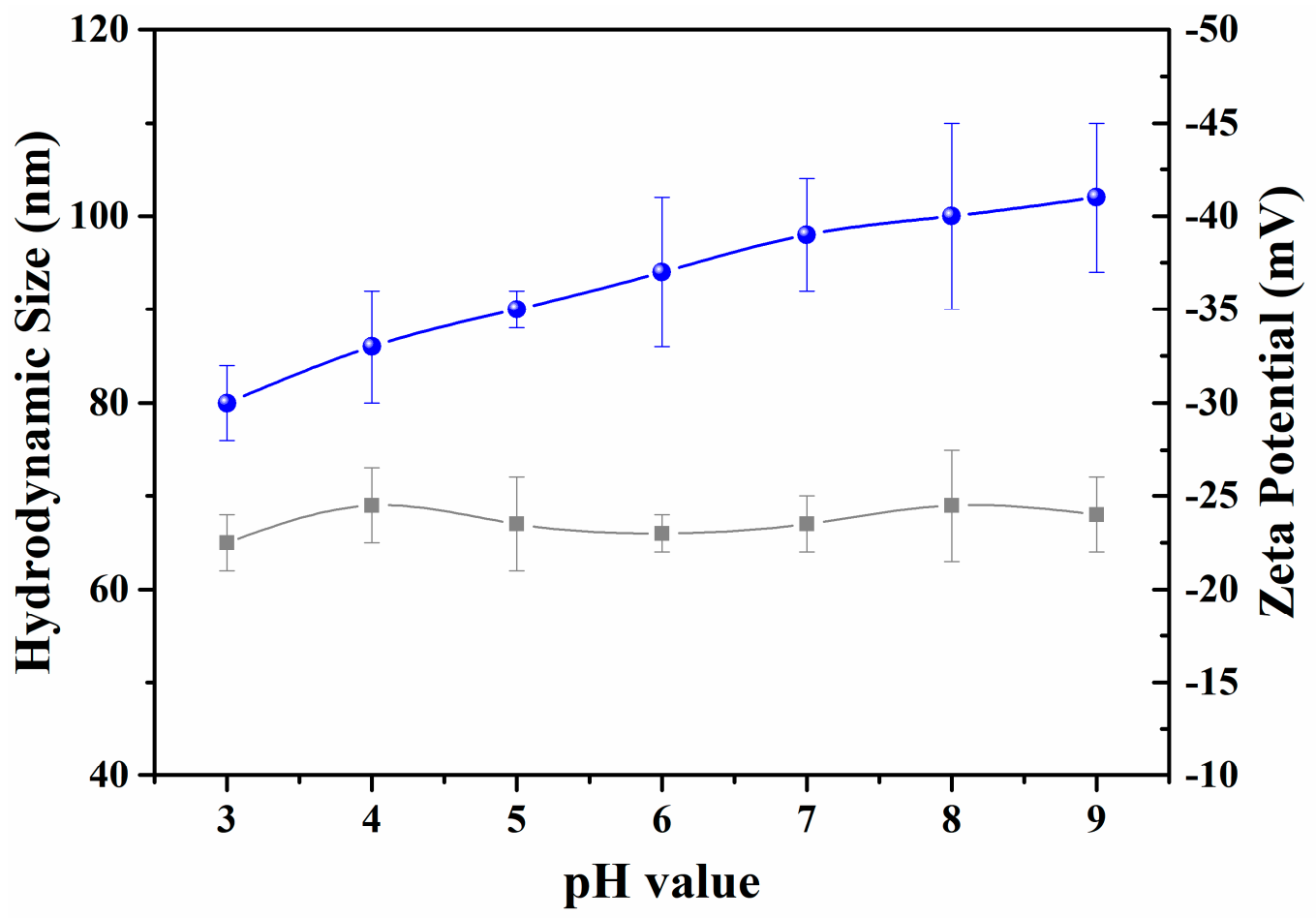

Figure 4. Variation of hydrodynamic size and zeta potential of LNPs with different $\mathrm{pH}$ values.
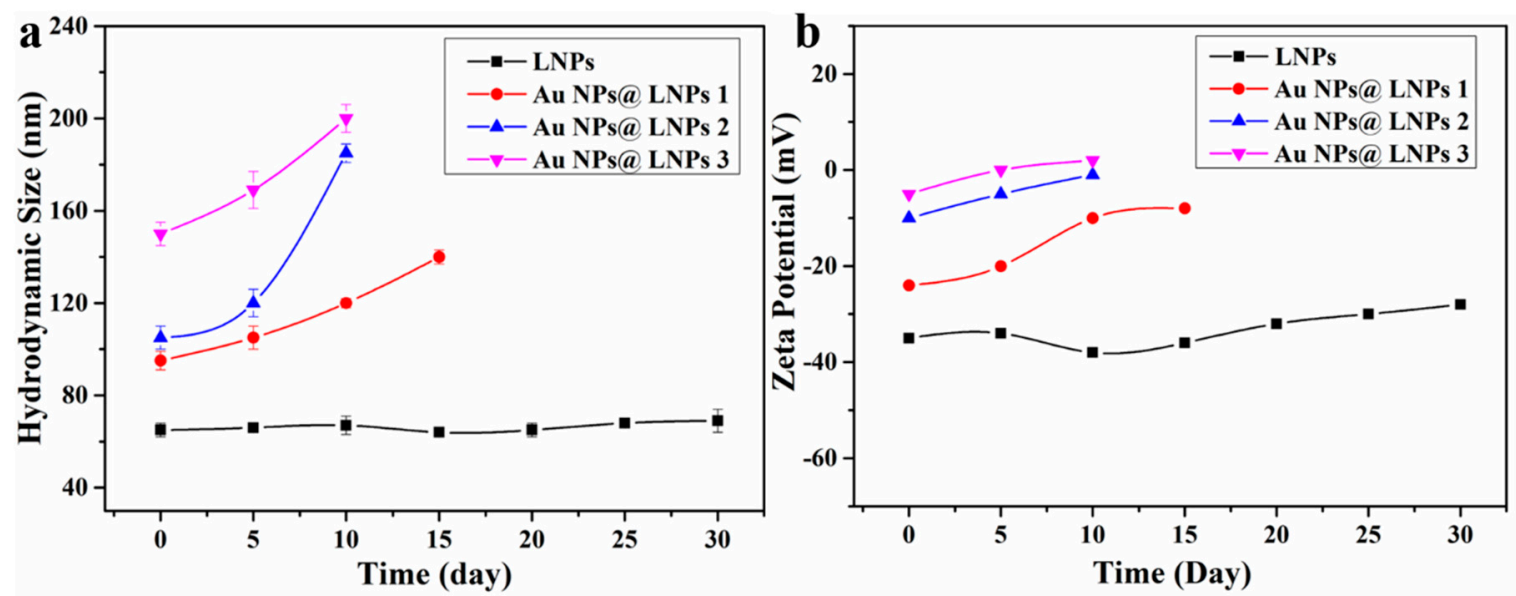

Figure 5. Variation of hydrodynamic size (a) and zeta potential (b) of LNPs and The composites during long-term storage.

\subsection{UV-Vis Spectrum of The Preparation of LNPs@AuNPs}

Lignin was utilized as a reducing agent and stabilizing agent for The preparation of Au NPs. LNPs enabled The well-ordered structure of The nanocomposites. UV-Vis spectra were used to monitor The preparation of Au NPs (Figure 6). The LNPs were formed due to hydrogen bonding, van der Waal's forces, electrostatic interaction, and $\pi-\pi$ interactions. The adsorption band at $280 \mathrm{~nm}$ is The characteristic band of lignin [23]. The adsorption of nanocomposites demonstrated a red shift indicating The interaction between metal ions and negatively charged LNPs. The LNPs@AuNPs showed The strong characteristic of The SPR adsorption band of Au NPs at $540 \mathrm{~nm}$ which confirmed The successful preparation of Au NPs (LNPs@AuNPs 1). With The increase in The concentration of $\mathrm{HAuCl}_{4}$, The characteristic adsorption band showed a blue shift, indicating that The size of The nanoparticles decreased (LNPs@AuNPs 2) [24]. 


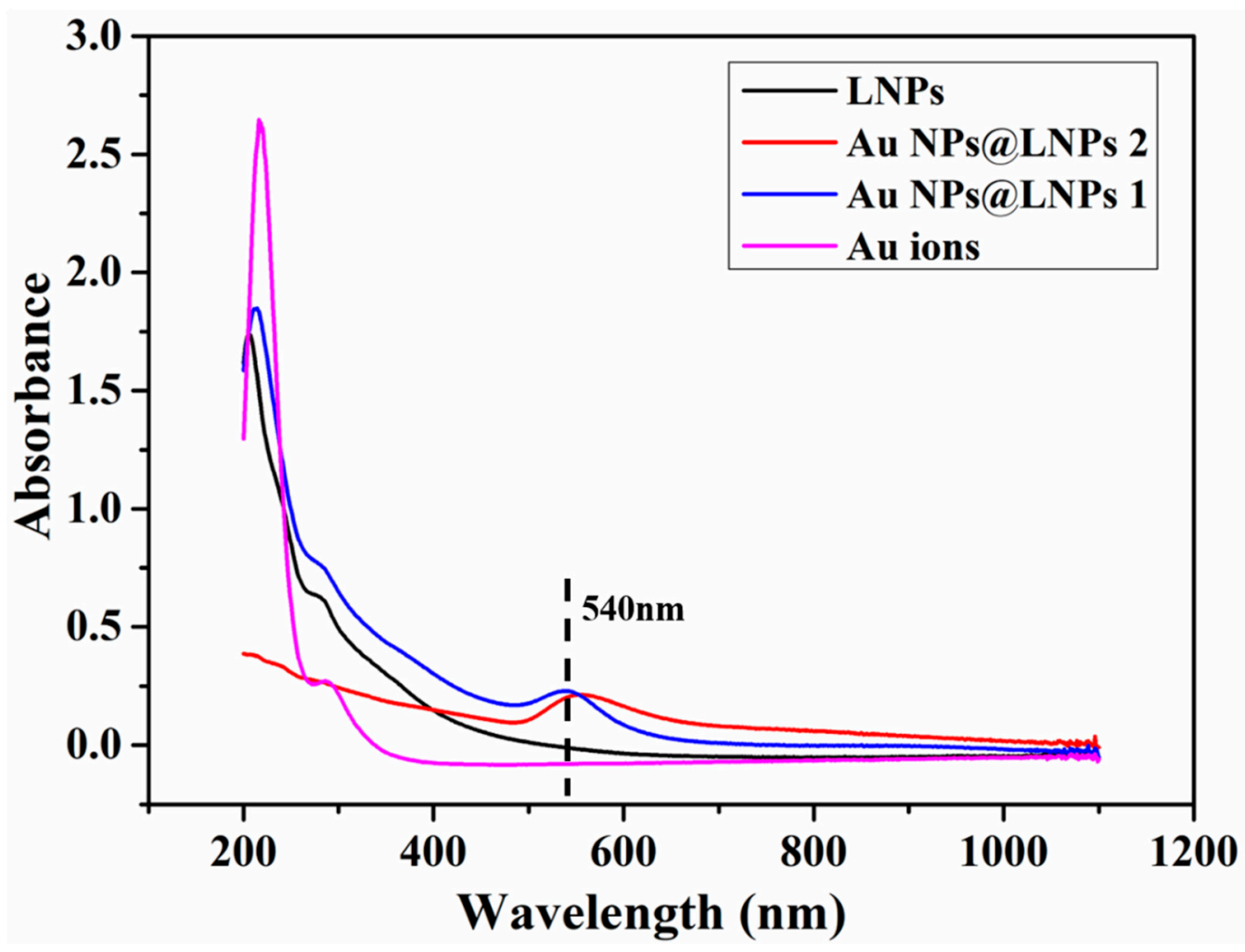

Figure 6. UV-Vis spectra of The LNPs, Au ions and LNPs@AuNPs.

\section{Discussion}

\subsection{TEM of LNPs and Au NPs@LNPs}

TEM was used to further verify The morphology of The LNPs and LNPs@AuNPs. The reduction process and morphology variation of The LNPs were monitored by TEM. Figure 7a,b shows The morphology of The LNPs. The LNPs demonstrated two main structures: yolk shell structure and hollow structure. According to statistics, The size of The yolk shell LNPs occupied 77\% of The total LNPs with a size below $70 \mathrm{~nm}$, while The hollow LNPs occupied 23\% of The total LNPs with a size between 80 and $100 \mathrm{~nm}$.

The Au NPs were synthesized in situ by using The hydroxyl and aldehyde groups of The LNPs. Figure 7c,d shows The morphology of The LNPs@AuNPs. The diameter of The gold nanoparticles was in The range of 5-30 nm. The well-defined structure of The LNPs and well-dispersed gold nanoparticles verified that LNPs could act as excellent reducing agents and capping agents for The preparation of gold nanoparticles. Figure $7 \mathrm{e}, \mathrm{f}$ demonstrates that The increased $\mathrm{HAuCl}_{4}$ concentration led to decreased average gold nanoparticles. However, The increased $\mathrm{HAuCl}_{4}$ concentration resulted in The collapse of The hollow structure of LNPs. Part of gold nanoparticles are from The LNPs. Thus, a moderate $\mathrm{HAuCl}_{4}$ concentration should be controlled. Figure $7 \mathrm{~g}$, h indicates The Au NPs prepared at $80{ }^{\circ} \mathrm{C}$ for $30 \mathrm{~min}$. The increased temperature led to The inferior control of The growth of gold nanoparticles. Furthermore, The Au NPs@ LNP composites exhibited excellent dispersal ability after storage for seven days (Figure 7i,j). These results verified that well-dispersed gold nanoparticles with well-defined lignin nanoparticles could be prepared at room temperature with The concentration of $\mathrm{HAuCl}_{4}$ at $0.5 \mathrm{mmol} / \mathrm{L}$. 

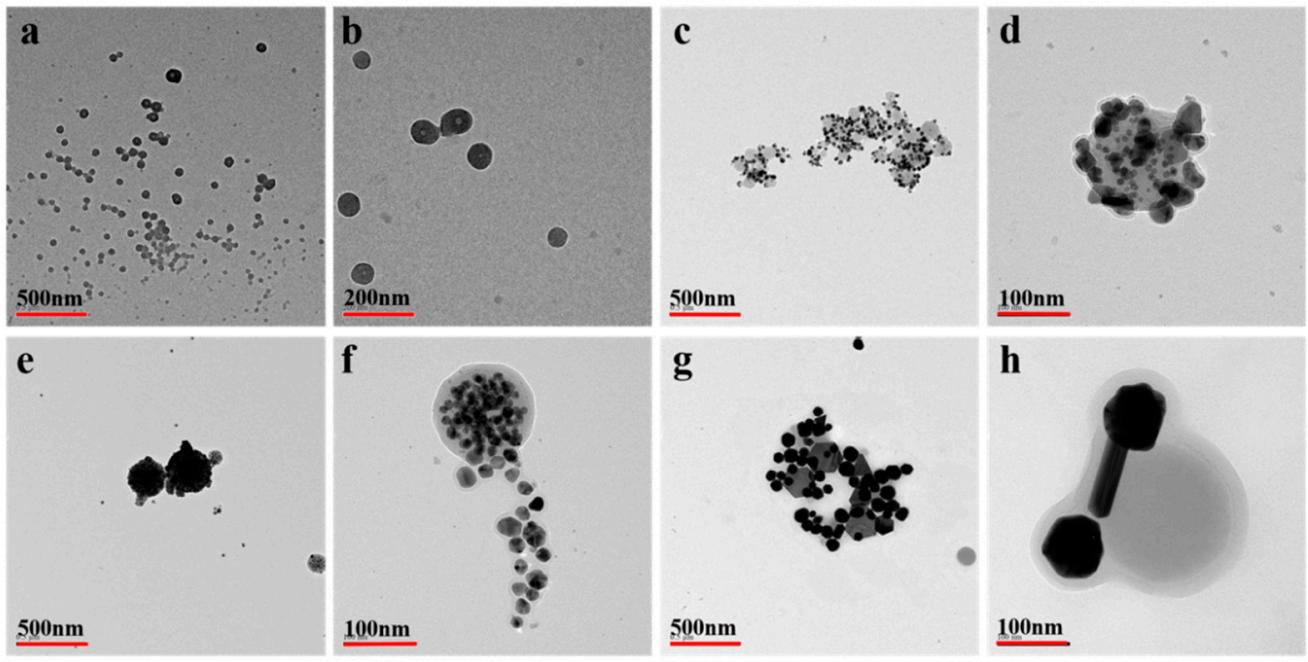

h
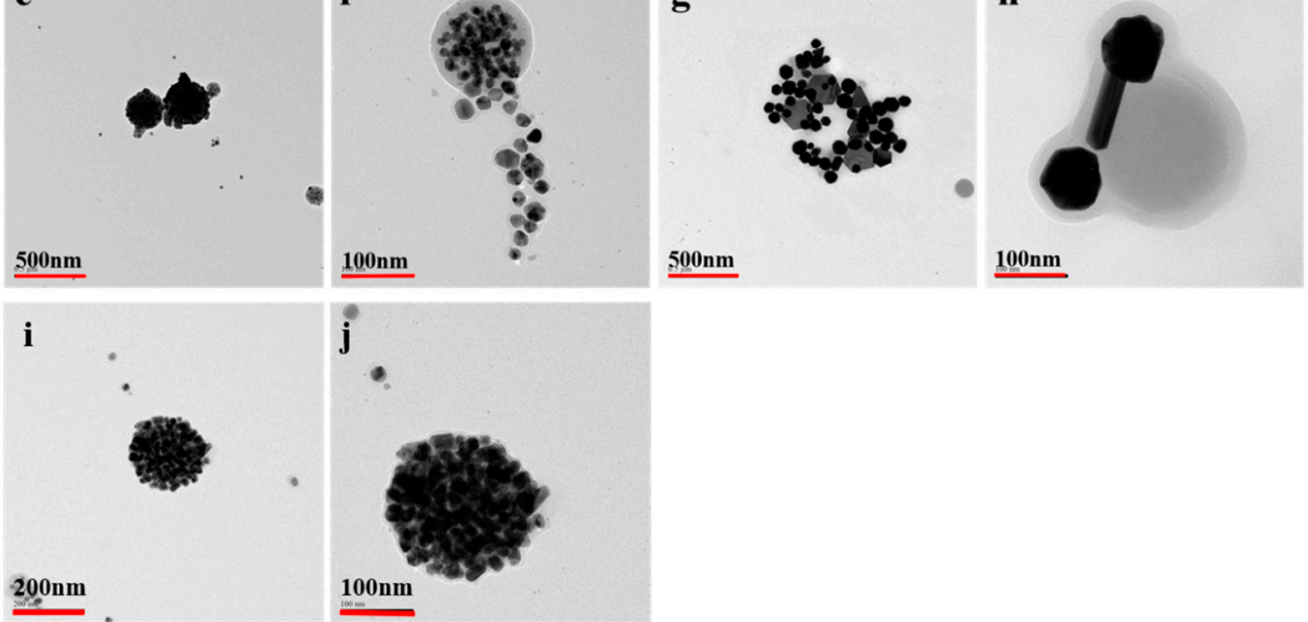

Figure 7. TEM of The LNPs (a,b), LNPs@AuNPs 1 (c,d), LNPs@AuNPs 2 (e,f), LNPs@AuNPs 3 (g,h), Au NPs@ LNPs 2 storage for seven days $(\mathbf{i}, \mathbf{j})$.

ICP-AES was used to quantify The loading of Au NPs onto The LNPs. The Au NP loading for LNPs@AuNPs 1, LNPs@AuNPs 2, and LNPs@AuNPs 3 was 4.59\%, 12.54\%, and 8.59\%, respectively. The loading of Au NPs increased with The increase in precursor concentration and temperature.

\subsection{XPS of LNPs and Au NPs@LNPs}

XPS wide scans showed The signals of carbon and oxygen (Figure 8a). The LNPs@AuNPs exhibited The signals of Au which further confirmed The successful preparation of gold nanoparticles (Figure 8b). The C 1s spectra of LNPs and LNPs@AuNPs with their deconvolutions are shown in Figure $8 \mathrm{c}, \mathrm{d}$, respectively. The deconvolution of LNPs and LNPs@AuNPs on C 1s shows four similar peaks: peak 1 with binding energy $284.7 \mathrm{eV}$ is related to a carbon bound to carbon atoms (C-C) or carbon-carbon double bond $(\mathrm{C}=\mathrm{C})$, peak 2 at $286.5 \mathrm{eV}$ corresponds to a carbon atom bound to a non-carbonyl oxygen atom $(\mathrm{C}-\mathrm{OH})$, peak 3 at $285.4 \mathrm{eV}$ represents a carbon atom bound to one carbonyl oxygen atom $(\mathrm{C}=\mathrm{O})$ or two non-carbonyl oxygen atoms $(\mathrm{O}-\mathrm{C}-\mathrm{O})$, and peak 4 at $288.6 \mathrm{eV}$ corresponds to peaks for $\mathrm{O}-\mathrm{C}=\mathrm{O}$. Compared with The LNPs, The bands signal of $\mathrm{C}=\mathrm{O}$ and $\mathrm{O}-\mathrm{C}=\mathrm{O}$ are stronger, while The signal of $\mathrm{C}-\mathrm{O}$ decreased obviously. Thus, The side chain of lignin was disrupted during The reduction process, and The quinone structure or carboxylic groups were formed [25]. 

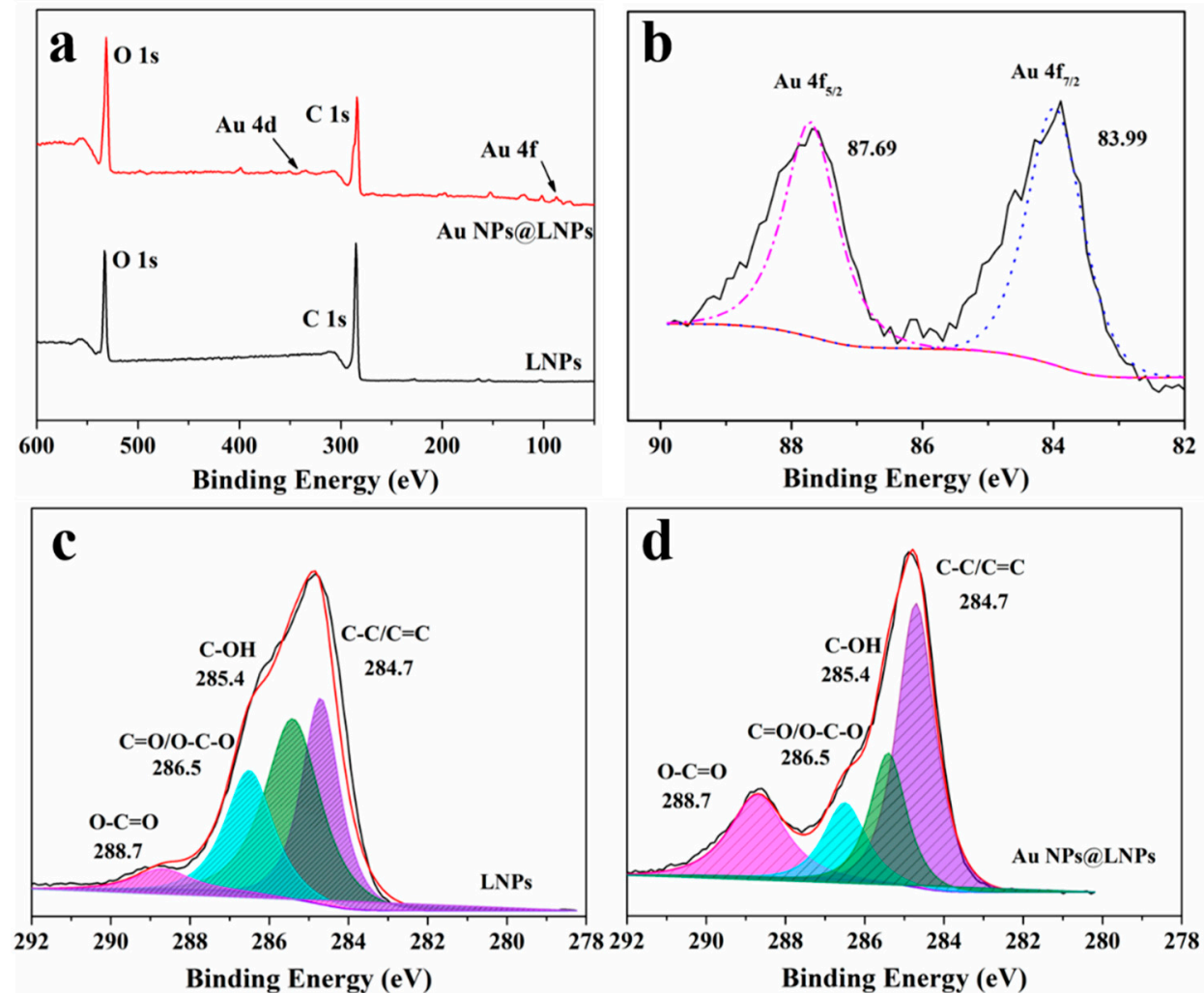

Figure 8. (a) XPS of The wide scan of LNPs and Au NPs@ LNPs. (b) Au 4f of The Au NPs@ LNPs (c) C 1s of The LNPs, (d) C 1s of The Au NPs@ LNPs.

\subsection{Proposed Mechanism for The Preparation of LNPs@AuNPs}

The green synthesis of gold nanoparticles is related to electrostatic interaction and The reduction effect due to hydroxyl and aldehyde groups in The lignin structure. Adsorption and reduction were completed first on The surface of The LNPs, and large metal nanoparticles formed. As The reaction proceeded, The $\mathrm{Au}^{3+}$ was adsorbed inside The LNPs, The gold nanoparticles formed slowly inside The LNPs, and The gold nanoparticles were small and well dispersed due to The stabilizing/capping effect.

Lignin contains stable radicals at room temperature. The main radicals have a semi-quinone structure [25]. During The reduction process, The hydroquinones and quinone structure of lignin formed due to The comproportionation reaction of semiquinone radicals, and semiquinone radical anions might be related to The formation of gold nanoparticles (Figure 9I). Also, The abundant phenolic groups favor The reduction of Au NPs. The surface hydroxyl groups would be transferred to carbonyl/carboxylic groups which contribute to The preparation of Au NPs (Figure 9II). 

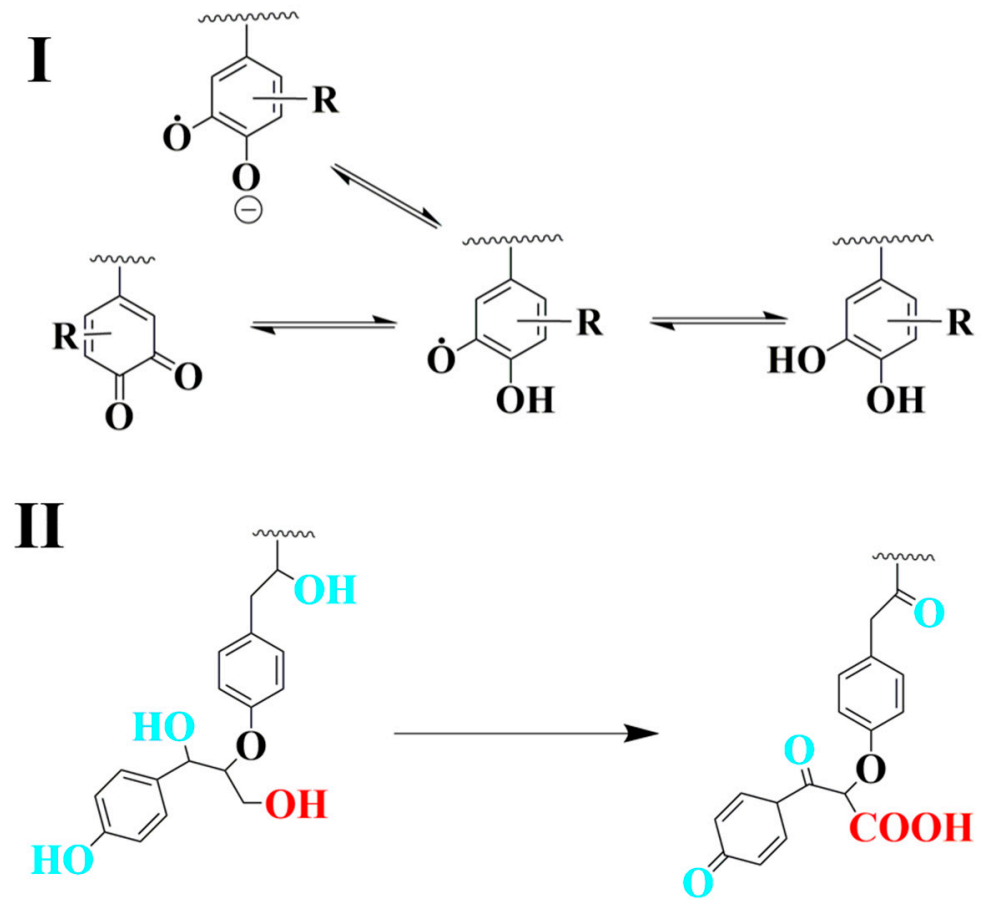

Figure 9. Proposed mechanism for The preparation of gold nanoparticles: (I) quinone related reduction mechanism, (II) hydroxyl groups related reduction mechanism.

\section{Conclusions}

To achieve total green synthesis of Au NPs with lignin, we reported The preparation of LNPs@AuNPs by using LNPs without chemical addition at room temperature. The LNPs acted as a reducing agent, stabilizing agent, and template for The preparation of LNPs@AuNP nanocomposites. The abundant noncondensed hydroxyl groups (11.49 mmol/g) favored The preparation of Au NPs. The Au NP diameters of 1-30 nm were well dispersed in The LNPs. The optimal reaction conditions were a ratio of $10 \mathrm{mg}$ of LNPs to $0.05 \mathrm{mmol} \mathrm{HAuCl}_{4}$, room temperature, and a reaction time of 30 min. The Au NPs@ LNPs exhibited excellent stability in The suspension for more than seven days. The reduction process could be related to (i) disruption of side chains of lignin, hydroquinones, and quinones from The comproportionation reaction, (ii) The surface hydroxyl groups would be transferred to carbonyl/carboxylic groups, and (iii) abundant noncondensed phenolic groups. The LNPs@AuNPs would inspire The design of LNPs@AuNPs derived from functional materials for application as sensors, responsive material, and actuators.

Author Contributions: Conceptualization, B.W. and G.Y.; methodology, B.W. and G.F.; software, B.W.; validation, B.W., G.Y. and G.F.; formal analysis, B.W.; investigation, J.C.; resources, J.C.; data curation, B.W.; writing-original draft preparation, B.W.; writing-review and editing, G.Y. and G.F.; visualization, G.Y.; supervision, G.Y.; project administration, G.Y.; funding acquisition, G.Y. All authors have read and agreed to The published version of the manuscript.

Funding: The authors are grateful for The financial support from The National Key Research and Development Program of China (Grant No. 2017YFB0307900), The National Natural Science Foundation of China (Grant No. 31770628), The Provincial Key Research and Development Program of Shandong (Grant No. 2019JZZY010326), and The Taishan Scholars Program.

Acknowledgments: The authors are grateful to Larry A. Calhoun and Steven Cogswell for their assistance in The analysis of TEM and ${ }^{31} \mathrm{P}$.

Conflicts of Interest: The authors declare no conflict of interest. 


\section{References}

1. Anker, J.N.; Hall, W.P.; Lyandres, O.; Shah, N.C.; Zhao, J.; Van Duyne, R.P. Biosensing with plasmonic nanosensors. Nat. Mater. 2008, 7, 442-453.

2. Kamat, P.V. Photophysical, Photochemical and Photocatalytic Aspects of Metal Nanoparticles. J. Phys. Chem. B 2002, 106, 7729-7744.

3. Chou, C.; Chen, C.; Wang, C.R.C. Highly efficient, wavelength-tunable, gold nanoparticle based optothermal nanoconvertors. J. Phys. Chem. B 2005, 109, 11135-11138.

4. Huang, P.; Lin, J.; Li, W.; Rong, P.; Wang, Z.; Wang, S.; Wang, X.; Sun, X.; Aronova, M.A.; Niu, G. Biodegradable Gold Nanovesicles with an Ultrastrong Plasmonic Coupling Effect for Photoacoustic Imaging and Photothermal Therapy. Angew. Chem. 2013, 52, 13958-13964.

5. Gaiduk, A.; Ruijgrok, P.V.; Yorulmaz, M.; Orrit, M. Making gold nanoparticles fluorescent for simultaneous absorption and fluorescence detection on The single particle level. Phys. Chem. Chem. Phys. 2011, 13, 149-153. [CrossRef]

6. $\quad$ Lee, S.M.; Kim, H.J.; Ha, Y.J.; Park, Y.N.; Lee, S.K.; Park, Y.; Yoo, K. Targeted chemo-photothermal treatments of rheumatoid arthritis using gold half-shell multifunctional nanoparticles. ACS Nano 2013, 7, 50-57.

7. Tangeysh, B.; Tibbetts, K.M.; Odhner, J.H.; Wayland, B.B.; Levis, R.J. Gold Nanoparticle Synthesis Using Spatially and Temporally Shaped Femtosecond Laser Pulses: Post-Irradiation Auto-Reduction of Aqueous [AuCl4]-. J. Phys. Chem. C 2013, 117, 18719-18727.

8. Lu, X.; Tuan, H.; Korgel, B.A.; Xia, Y. Facile synthesis of gold nanoparticles with narrow size distribution by using $\mathrm{AuCl}$ or $\mathrm{AuBr}$ as The precursor. Chem. Eur. J. 2008, 14, 1584-1591.

9. Soliwoda, K.; Rosowski, M.; Tomaszewska, E.; Tkaczszczesna, B.; Celichowski, G.; Psarski, M.; Grobelny, J. Synthesis of monodisperse gold nanoparticles via electrospray-assisted chemical reduction method in cyclohexane. Colloids Surf. A Physicochem. Eng. Asp. 2015, 482, 148-153.

10. Huang, H.; Yang, X. Synthesis of polysaccharide-stabilized gold and silver nanoparticles: A green method. Carbohydr. Res. 2004, 339, 2627-2631.

11. Coccia, F.; Tonucci, L.; Bosco, D.; Bressan, M.; Dalessandro, N. One-pot synthesis of lignin-stabilised platinum and palladium nanoparticles and their catalytic behaviour in oxidation and reduction reactions. Green Chem. 2012, 14, 1073-1078.

12. Ratanasumarn, N.; Chitprasert, P. Cosmetic potential of lignin extracts from alkaline-treated sugarcane bagasse: Optimization of extraction conditions using response surface methodology. Int. J. Biol. Macromol. 2020, 153, 138-145. [CrossRef]

13. Tarasov, D.; Leitch, M.; Fatehi, P. Lignin-carbohydrate complexes: Properties, applications, analyses, and methods of extraction: A review. Biotechnol. Biofuels 2018, 11, 269. [CrossRef]

14. Han, G.; Wang, X.; Hamel, J.; Zhu, H.; Sun, R. Lignin-AuNPs liquid marble for remotely-controllable detection of Pb2+. Sci. Rep. 2016, 6, 38164. [CrossRef]

15. Lin, X.; Zhao, J.; Wu, M.; Kuga, S.; Huang, Y. Green Synthesis of Gold, Platinum and Palladium Nanoparticles by Ligninand Hemicellulose. Res. Rev. J. Microbiol. Biotechnol. 2016, 5, 14-18.

16. Chatterjee, S.; Saito, T. Lignin-derived advanced carbon materials. ChemSusChem 2015, 8, 3941-3958.

17. Chen, L.; Shi, Y.; Gao, B.; Zhao, Y.; Jiang, Y.; Zha, Z.; Xue, W.; Gong, L. Lignin Nanoparticles: Green Synthesis in a $\gamma$-Valerolactone/Water Binary Solvent and Application to Enhance Antimicrobial Activity of Essential Oils. ACS Sustain. Chem. Eng. 2019, 8, 714-722. [CrossRef]

18. Rangan, A.; Manjula, M.; Satyanarayana, K.; Menon, R. Lignin/nanolignin and their biodegradable composites. In Biodegradable Green Composites; Wiley Online Library: Hoboken, NJ, USA, 2016; pp. 167-198.

19. Dai, L.; Li, Y.; Kong, F.; Liu, K.; Si, C.; Ni, Y. Lignin-Based Nanoparticles Stabilized Pickering Emulsion for Stability Improvement and Thermal-Controlled Release of trans-Resveratrol. ACS Sustain. Chem. Eng. 2019, 7, 13497-13504. [CrossRef]

20. Chen, S.; Wang, G.; Sui, W.; Parvez, A.M.; Dai, L.; Si, C. Novel lignin-based phenolic nanosphere supported palladium nanoparticles with highly efficient catalytic performance and good reusability. Ind. Crop. Prod. 2020, 145, 112164. [CrossRef]

21. Ma, M.; Dai, L.; Si, C.; Hui, L.; Liu, Z.; Ni, Y. A facile approach to prepare super long-term stable lignin nanoparticles from black liquor. ChemSusChem 2019, 12, 5239. [CrossRef] 
22. Shen, Z.; Luo, Y.; Wang, Q.; Wang, X.; Sun, R. High-Value Utilization of Lignin to Synthesize Ag Nanoparticles with Detection Capacity for Hg2+. ACS Appl. Mater. Interfaces 2014, 6, 16147-16155. [CrossRef]

23. Deng, Y.; Feng, X.; Zhou, M.; Qian, Y.; Yu, H.; Qiu, X. Investigation of Aggregation and Assembly of Alkali Lignin Using Iodine as a Probe. Biomacromolecules 2011, 12, 1116-1125. [CrossRef]

24. Martinez, J.C.; Chequer, N.A.; Gonzalez, J.L.; Cordova, T. Alternative Metodology for Gold Nanoparticles Diameter Characterization Using PCA Technique and UV-VIS Spectrophotometry. Nanosci. Nanotechnol. 2013, 2, 184-189.

25. Bährle, C.; Nick, T.U.; Bennati, M.; Jeschke, G.; Vogel, F. High-Field Electron Paramagnetic Resonance and Density Functional Theory Study of Stable Organic Radicals in Lignin: Influence of The Extraction Process, Botanical Origin, and Protonation Reactions on The Radical g Tensor. J. Phys. Chem. A 2015, 119, 6475-6482. [CrossRef]

(C) 2020 by the authors. Licensee MDPI, Basel, Switzerland. This article is an open access article distributed under the terms and conditions of the Creative Commons Attribution (CC BY) license (http://creativecommons.org/licenses/by/4.0/). 